\title{
Radiation-Pattern Improvement of Patch Antennas Using a Compact Soft/Hard Surface (SHS) Structure on LTCC Multilayer Technology
}

\author{
G. DeJean, R. L. Li, M. M. Tentzeris, J. Papapolymerou, and J. Laskar \\ School of Electrical and Computer Engineering \\ Georgia Institute of Technology, Atlanta, GA 30332-0250, USA
}

\begin{abstract}
A compact soft/hard surface (SHS) structure consisting of a square ring of shortcircuited metal strips is employed to surround the patch antenna for blocking the surfacewave propagation, thus alleviating the effect of the edge diffraction and hence improving the radiation pattern. The operating frequency of the SHS is determined by the width of the metal strip, therefore it is suitable for a substrate with arbitrary thickness and dielectric constant. The compact SHS structure is realized on a low temperature co-fired ceramics (LTCC) technology. It is shown that the gain of a patch antenna can be increased to near 9 $\mathrm{dBi}$ through the use of the proposed compact SHS structure.
\end{abstract}

\section{INTRODUCTION}

Nowadays there is an increasing demand in wireless systems for integration of antennas with RF front-end circuits or even with IF and base-band components [1]. For highfrequency (such as millimeter-wave) applications, the size of a patch antenna may be much smaller compared to the printed circuit board (PCB) substrate, where the analog and digital devices are integrated [2]. When a patch antenna is fabricated on a larger-size substrate, its radiation pattern may be considerably affected by the diffraction of surface waves at the edge of the finite grounded substrate [3]. On the other hand, the excitation of strong surface waves also causes unwanted coupling between the antenna and other components on the PCB, thus degrading the performance of the integrated module [4].

The most popular technique for the improvement of the radiation performance of patch antennas on a large-size substrate is to construct an artificial periodic structure, such as photonic bandgap (PBG) or electromagnetic bandgap (EBG) [5], [6], surrounding the patch antenna to prevent the surface waves from propagating in the substrate. Unfortunately, it requires a considerable area to form a complete band gap structure. Another approach considered has been to use the concept of artificial soft/hard surface (SHS) for the improvement of the radiation patterns [7]. However, the conventional corrugation-based SHS structures require a substrate thickness of one quarter guided wavelength as well as a quite large area. Recently, a modern realization of artificial SHS structures has been described in [8]. The new proposed SHS consists of a number of quarter-wavelength metal strips that are short-circuited to the ground plane. The operating frequency for this SHS is determined by the strip width, not by the thickness of the substrate, allowing for its implementation in arbitrary substrate thicknesses. In this paper, we apply the concept of the modern SHS structure to improve the radiation pattern of patch antennas. A single square ring of the shorted quarter-wavelength metal strips is employed to surround the patch antenna for the suppression of outward propagating surface waves, thus alleviating the diffraction at the edge of the substrate. The effectiveness of the compact SHS structure in terms of radiation pattern improvement is verified by its implementation on LTCC multilayer technology.

\section{DESIGN OF THE COMPACT SHS STRUCTURE}

Consider a probe-fed square patch antenna on a square grounded substrate with thickness $H$ and a dielectric constant $\varepsilon_{\mathrm{r}}$. The patch antenna is surrounded by a square compact SHS 
structure, as shown in Fig. 1. The substrate thickness has a lateral size of $\mathrm{L} \times \mathrm{L}$, much larger than the size the square patch $\left(\mathrm{L}_{\mathrm{p}} \times \mathrm{L}_{\mathrm{p}}\right)$. The ideal compact SHS structure consists of a square ring of metal strips that are short-circuited to the ground plane by a metal wall along the outer edge of the ring. The inner length of the SHS ring (denoted by $L_{s}$ ) was found to be approximately one wavelength plus $L_{p}$. The width of the metal strip $\left(W_{s}\right)$ is approximately equal to a quarter of the guided wavelength.

\section{IMPLEMENTATION OF THE SHS STRUCTURE ON LTCC TECHNOLOGY}

To demonstrate the feasibility of the LTCC technology on the implementation of the SHS, we simulated a benchmarking prototype which is shown in Fig. 2. Note that the LTCC substrate is hidden in order to see the SHS structure clearly. The shorting wall of the SHS was replaced on the LTCC process with a ring of vias. The utilized LTCC material had a dielectric constant of 5.4. The whole module consists of a total of 11 LTCC layers (layer thickness $=100 \mu \mathrm{m}$ ) and 12 metal layers. To support the vias, on each metal layer a metal pad is required. To simplify the simulation, all pads on each metal layer are connected by a metal strip with a width of $600 \mu \mathrm{m}$. The size of the LTCC board was $30 \mathrm{~mm} \times 30 \mathrm{~mm}$. The design frequency is $16.5 \mathrm{GHz}$. The optimized values for $\mathrm{L}_{\mathrm{s}}$ and $\mathrm{W}_{\mathrm{s}}$ were respectively $22.2 \mathrm{~mm}$ and $1.4 \mathrm{~mm}$. Fifty vias on each side of the SHS ring were assigned. The total metal strip width for the SHS ring was found to be $1.7 \mathrm{~mm}$. A stacked configuration was adopted for the patch antenna to improve its input impedance performance. The upper and lower patches $(3.4 \mathrm{~mm} \times 3.4 \mathrm{~mm})$ were respectively printed on the first LTCC layer and the seventh layer from top. The lower patch was connected by a via hole to a microstrip line that is on the bottom surface of the LTCC substrate. The ground plane was embedded between the second and third LTCC layers from the bottom.

The simulated 3D radiation patterns of the stacked-patch antennas with and without the SHS are shown in Fig. 3. It is observed that there is a deep drop in the z-direction for the antenna without SHS due to the diffraction of surface waves at the edge of the substrate. The degradation of radiation pattern is significantly improved by the via-incorporated SHS. The radiation patterns measured in the E-plane are compared with simulated results in Fig. 4. We can see good agreement for the co-polarized components. It is confirmed that the radiation at broadside is enhanced and the backside level is reduced. Also the beamwidth in the E-plane is significantly reduced by the SHS. The maximum gain measured for the patch with the SHS is near $9 \mathrm{dBi}$, about $3 \mathrm{~dB}$ higher than the maximum gain and $7 \mathrm{~dB}$ higher than the gain at broadside for the antenna without the SHS.

\section{CONCLUSION}

The effectiveness of a modern SHS concept on the radiation-pattern improvement of a patch antenna on a large-size substrate has been investigated. The new SHS structure consists of a ring of short-circuited metal strips with a strip width of a quarter guided wavelength, much more compact than typical PBG/EBG structures. The compact SHS structure can be easily implemented in 3D modules, such as those fabricated using LTCC technology. Experimental results show that it is feasible to make use of the viametallization process on the LTCC technique for realization of a shorting wall required by the compact SHS structure. A maximum gain of near $9 \mathrm{dBi}$ can be achieved for the LTCC module.

\section{Acknowledgement}

The authors wish to acknowledge the support of Georgia Electronics Design Center, the NSF CAREER Award under contract NSF \#9964761, the NASA project under contract \#NCC3-1015, the NSF Award NSF ECS-0313951, and the NSF Packaging Research Center. 


\section{References}

[1] K. K. O, K. Kim, and et al, "Wireless communications using integrated antenna," Proceedings of the IEEE Interconnect Technology Conference, pp. 111-113, June 2003.

[2] J.-Y. Park, S.-S. Jeon, Y. Wang. And T. Itoh, "Integrated antenna with direct conversion circuitry for broad-band millimeter-wave communications," IEEE Trans. Microwave Theory Tech., vol. 51, no. 5, pp. 1482-1488, May 2003.

[3] S. Maci, L.Borelli, and L. Rossi, "Diffraction at the edge of a truncated grounded dielectric slab,” IEEE Trans. Antennas Propagat., vol. 44, pp. 863-872, June 1996.

[4] D. Moongilan, "Grounding optimization techniques for controlling radiation and crosstalk in mixed signal PCBs," Proceedings of IEEE Electromagnetic Compatibility Symposium, vol. 1, pp. 495-500. Aug. 1998.

[5] R. Gonzalo, P. de. Maagt, and M. Sorolla, "Enhanced patch-antenna performance by suppressing surface waves using photonic-bandgap substrates," IEEE Trans. Microwave Theory Tech., vol. 47, no. 11, pp. 2131-2138, Nov. 1999.

[6] R. Coccioli, F.-R. Yang, K.-P. Ma, and T. Itoh, "Aperture-coupled patch antenna on UCPBG substrate,” IEEE Trans. Microwave Theory Tech., vol. 47, no. 11, pp. 2123-2130, Nov. 1999.

[7] Z. Ying and P.-S. Kildal, "Improvements of dipole, helix, spiral, microstrip patch and aperture antennas with ground planes by using corrugated soft surfaces," IEE Proc.-Microw. Antennas Propag., vol. 143, no. 3, pp. 244-248, 1996.

[8] G. Ruvio, P.-S. Kildal, and S. Maci, "Modal propagation in ideal soft and hard waveguides," Proceedings of IEEE Antenna and Propagation Society International Symposium, vol. 4, pp. 438-441, June 2003.

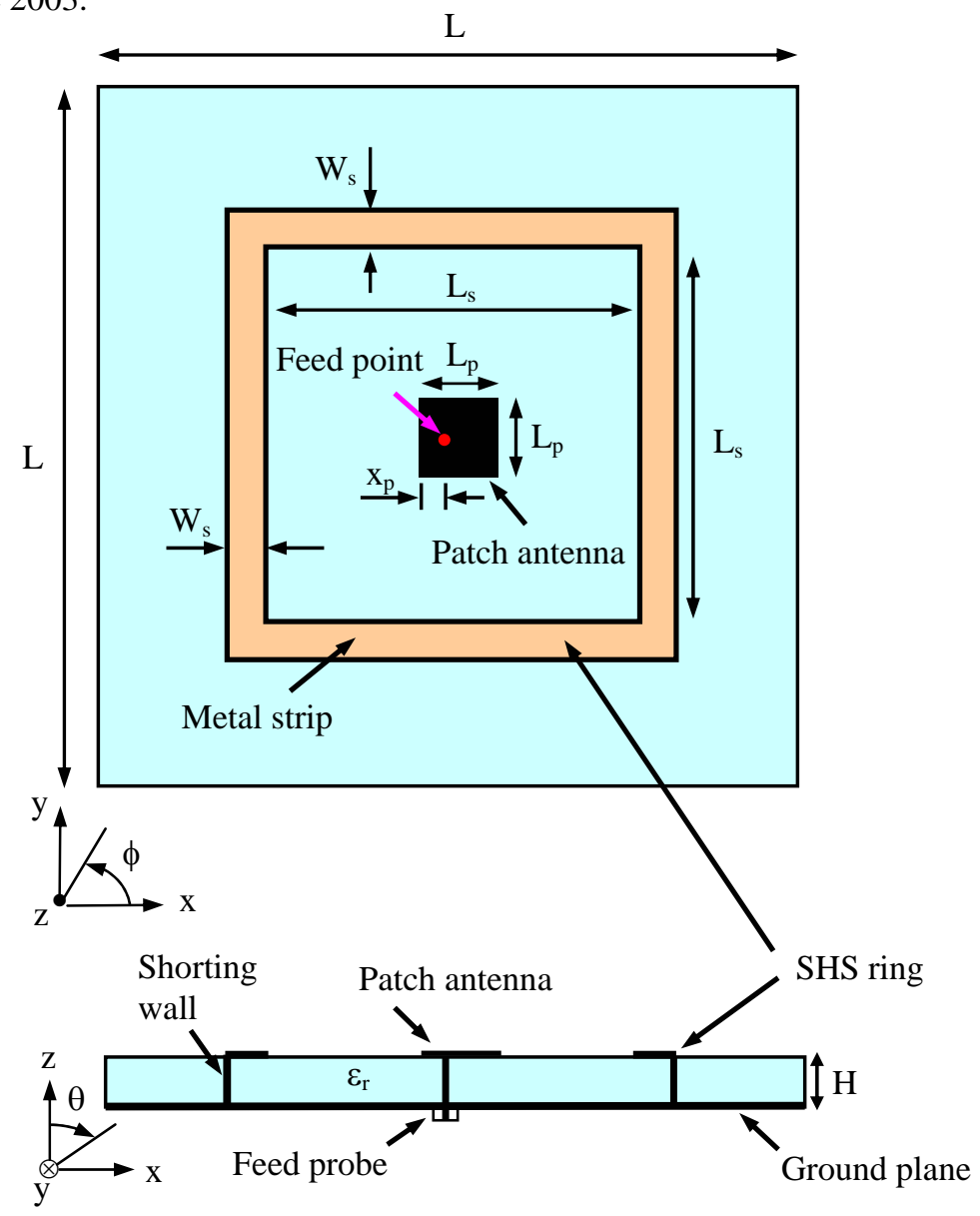

Fig. 1. Patch antenna surrounded by an ideal compact SHS structure. 


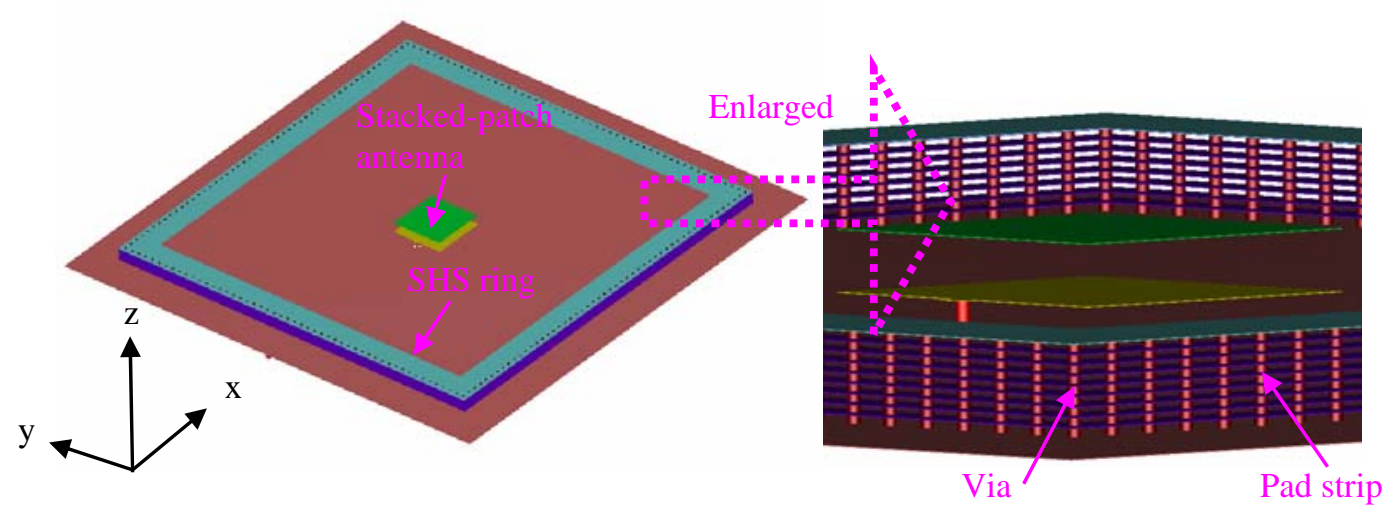

Fig. 2. Patch antenna surrounded by a compact SHS structure on LTCC technology.

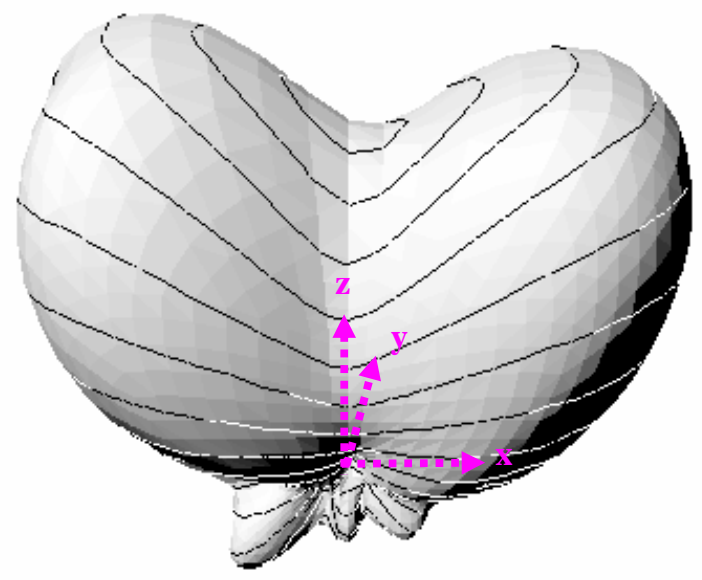

(a) Without SHS

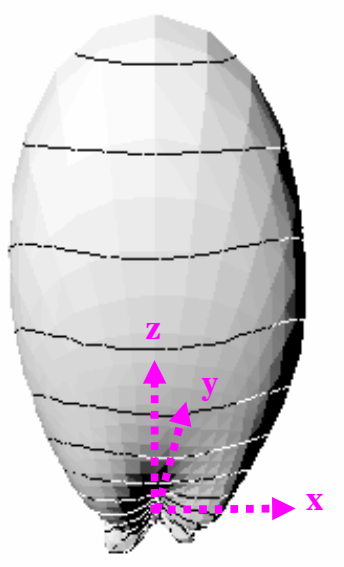

(b) With SHS

Fig. 3. 3D radiation patterns of the patch antennas with and without the SHS.

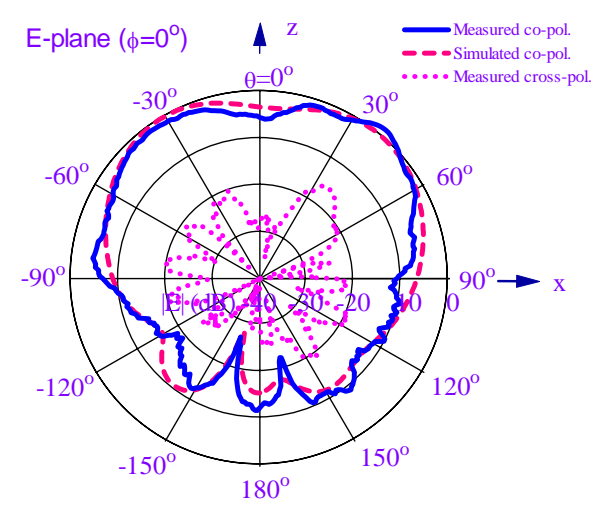

(a) Without SHS

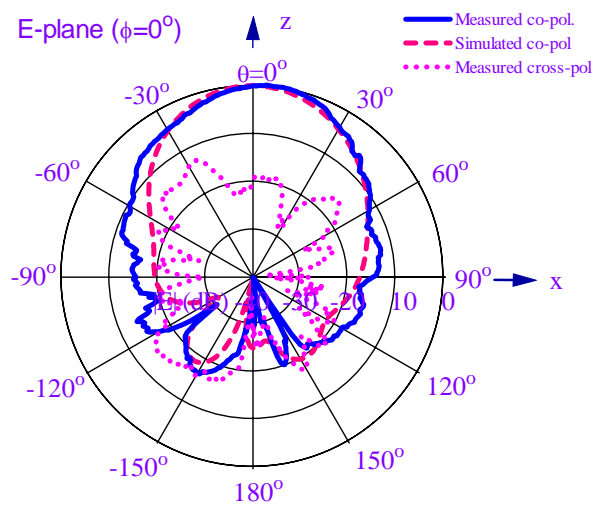

(b) With SHS

Fig. 4. Radiation patterns in E-plane for the patch antennas with and without the SHS. 